\title{
Fluid-structure simulation of a viscoelastic hydrofoil subjected to quasi-steady flow
}

\author{
R. L. Campbell, E. G. Paterson, M. C. Reese \& S. A. Hambric \\ Penn State Applied Research Laboratory, USA
}

\begin{abstract}
Fluid-structure interaction simulations are performed for a flexible hydrofoil subjected to quasi-steady flow conditions. The hydrofoil is fabricated from a polymeric material that exhibits viscoelastic effects, causing the hydrofoil to change shape while subjected to the fluid loads. The time-dependent deformations and loads will be compared in the future to empirical results from upcoming water tunnel tests. The fluid-structure interaction simulations are performed using a tightly coupled partitioned approach, with OpenFOAM as the flow solver and a finite element solver for the structural response. The codes are coupled using a fixed-point iteration with relaxation. The flow is modeled as laminar and quasisteady. Simulations indicate the hydrofoil angle of attack (AOA) changes from zero to a negative value as the material relaxes. The approach used here is being developed for application to a blood pump that has a performance closely tied to blade deformation through the impeller tip clearance.

Keywords: fluid-structure interaction, viscoelasticity, hydrofoil, OpenFOAM.
\end{abstract}

\section{Introduction}

The area of fluid-structure interaction (FSI) modeling has been very active in recent years, as evidenced by the numerous papers in the literature (see, for example, the review by Tezduyar and Sathe [1]). The dramatic advances in affordable computational resources coupled with improved modeling capability for both fluid and solid structures over the past two decades have made feasible coupled fluid/solid simulations for real-world engineering applications. While FSI simulations have been an area of research since the late 1970s (see Felippa et al. [2]), there still exist several challenges before these simulations will be 
routinely applied to real-world engineering applications (Tezduyar and Sathe [1] and Longatte et al. [3]).

The present application is related to a flexible blood pump impeller with a tip clearance (i.e., the clearance between the blade tips and the pump housing) that is very sensitive to blade deflections. The impeller is fabricated from a polymer that has a time-dependent response to applied loads (creep) and enforced displacements (stress relaxation), and therefore requires the use of a viscoelastic material model. The influence of blade deformation on pump performance is expected to be very strong because of the anticipated tip gap changes.

The approach to solving this problem involves several steps. The first step is to implement a FSI solver capable of modeling the nonlinear, time dependent polymeric material behaviour. Verification and validation of this solver is the second step and will be performed using a simple hydrofoil constructed of the same polymer as the blood pump impeller, and subjected to flow in a water tunnel for which net blade loads and blade deformation will be measured. The modeling approach will then be modified and updated as necessary and then applied to the actual rotating impeller of the blood pump. Time-dependent performance predictions of the pump will be compared to empirical results. The focus of the current paper is the fluid-structure simulations of the simple polymeric hydrofoil operating in the water tunnel test section.

\section{Problem description}

A single hydrofoil constructed of a polymeric material is affixed in a water tunnel test section with zero angle of attack (AOA). The hydrofoil is a modified NACA series 66 with $a=0.8$ camber [4] and thus has nonzero lift at zero AOA. The upstream flow boundary is modeled as a uniform inflow with a velocity of $2 \mathrm{~m} / \mathrm{s}$ and the downstream flow boundary is modeled with a zero pressure condition. The no-slip condition is applied at all other fluid boundaries. The foil is constrained at the root and is subjected to fluid pressures at the fluid/solid interface, $\Gamma_{F / S}$. A schematic of the setup is shown in figure 1. The test section has a length (L) of $0.762 \mathrm{~m}$, width $(\mathrm{W})$ of $0.508 \mathrm{~m}$, and height $(\mathrm{H})$ of $0.114 \mathrm{~m}$. The distance from the inlet to the foil leading edge is $0.356 \mathrm{~m}$. The foil has a chord length (c) of $0.050 \mathrm{~m}$ and a span (s) of $0.100 \mathrm{~m}$.

The Reynolds number, based on hydrofoil chord, for this flow is approximately 100,000 and the critical Reynolds number for transition to turbulence is on the order of 200,000. Therefore, it is reasonable to employ a laminar flow model for the first attempt at modeling this problem.

\section{Fluid-structure simulation approach}

The governing equations for continuum mechanics (both fluids and solids), cast in an arbitrary Lagrangian Eulerian (ALE) form, are as follows. Mass conservation 


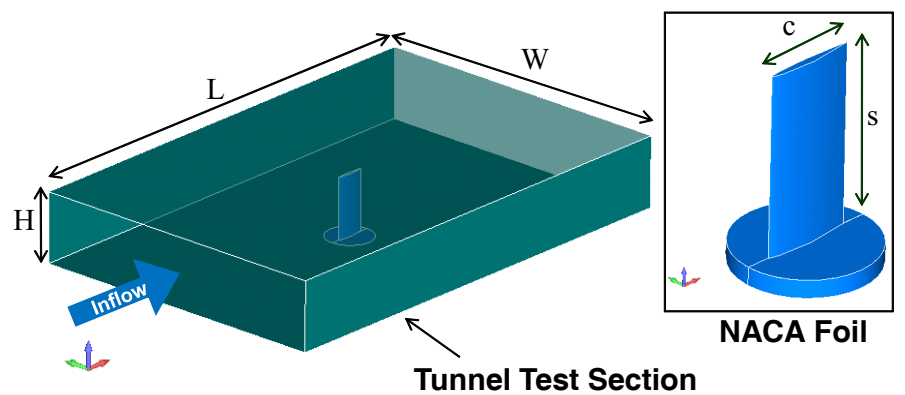

Figure 1: Flow over a cantilevered modified NACA 66 hydrofoil with uniform inflow conditions.

is governed by the continuity equation:

$$
\frac{\partial \rho}{\partial t}+\nabla \cdot\left[\rho\left(\boldsymbol{v}-\boldsymbol{v}^{a l e}\right)\right]=0,
$$

where $\rho$ is mass density, $\boldsymbol{v}$ is the particle velocity, and $\boldsymbol{v}^{\text {ale }}$ is the grid point velocity. For a Lagrangian implementation, $\boldsymbol{v}^{\text {ale }}=\boldsymbol{v}$, and for an Eulerian implementation, $\boldsymbol{v}^{\text {ale }}=0$. Performing a force balance and making use of the continuity equation leads to the following momentum equations:

$$
\rho \frac{\partial \boldsymbol{v}}{\partial t}+\rho\left[\left(\boldsymbol{v}-\boldsymbol{v}^{a l e}\right) \cdot \nabla\right] \boldsymbol{v}=\nabla \cdot \boldsymbol{\sigma}+\rho \boldsymbol{b},
$$

where $\sigma$ is the stress tensor and $\boldsymbol{b}$ is the body force.

The current effort makes use of a segregated approach, wherein each domain is modeled separately and employs different solvers. The fluid domain solver for this problem employs a finite volume discretization while the solid domain solver uses a finite element discretization. The use of a segregated approach enables the governing equations to be cast in different primitive variables: velocity for the fluid solver and displacement for the structure, as is traditional for each of these domains.

Because the fluid and solid time scales are much shorter than the viscoelastic relaxation (discussed below in Section 3.2) timescale, a quasi-steady simulation is used in this work. It is therefore not necessary to include the temporal components in the continuum equations shown above, but they are included for the sake of completeness. Note, however, that the solution time does play a role in the viscoelastic material model response.

While the governing equations for each domain (eqns (1) and (2)) are identical, the constitutive relationships required for closure of the equations differ, as described below along with the coupling requirements at the fluid/solid interface. 


\subsection{Flow solver}

OpenFOAM is the flow solver of choice for this effort because it facilitates custom integration with third-party solvers, has a pre-existing, robust mesh motion capability, and it is freely available through the GNU General Public License. The automatic mesh motion solver for the current problem makes use of a variable diffusion coefficient with quadratic dependence on the distance from the moving boundary (i.e., $\gamma=1 / l^{2}$ ), see Jasak and Tukovic [5]. The flow for this problem is approximated as incompressible and laminar, and the stress-strain closure is modeled as Newtonian:

$$
\boldsymbol{\sigma}=-p \boldsymbol{I}+2 \mu \boldsymbol{S}
$$

where $p$ is the thermodynamic pressure, $\mu$ is the absolute viscosity, $\boldsymbol{I}$ is the second rank unity tensor, and $S$ is the strain-rate tensor.

\subsection{Structural solver and solid model}

The structural solver employed for this work uses a Lagrangian finite element (FE) implementation, which means the mesh velocity is equivalent to the material velocity, $\boldsymbol{v}^{\text {ale }}=\boldsymbol{v}$. The momentum equation (eqn 2 ) becomes:

$$
\rho \frac{\partial^{2} \boldsymbol{u}}{\partial t^{2}}=\nabla \cdot \boldsymbol{\sigma}+\rho \boldsymbol{b},
$$

where $\boldsymbol{u}$ are the material displacements.

One of the important aspects of the current problem is the time dependency of the polymeric material. The time dependency is a result of a viscous-like material behaviour. The material also exhibits elasticity in that it will not continue to flow unbounded with the application of a finite stress. The viscoelasticity model employed for this work follows an approach similar to that used by the commercial software Abaqus (see the Abaqus User's Manual [6]) and derived in a similar manner by Kaliske and Rothert [7]. This model is for linear viscoelastic materials (which does not mean the time response of the material is linear, but rather the stress is proportional to strain at any given time: $\varepsilon[c \sigma(t)]=c \varepsilon[\sigma(t)]$, where $c$ is a constant) and uses the approximation that shear and volumetric behavior are independent. Experimental evaluation of the current material suggests that only the shear terms need to be modified by the viscoelastic model, which is consistent with most material behavior as reported by Kaliske and Rothert [7] and also in the Abaqus Manual [6].

The underlying material model is that of the Generalized Maxwell Element, which consists of Maxwell elements (i.e., a spring and dashpot in series) in parallel with a Hooke element (i.e., a spring) as shown in figure 2. The spring stiffness shown in this figure represents the material stiffness at infinite time (i.e., after all of the viscoelastic forces have diminished to zero). Each of the Maxwell elements are represented by a term in a Prony series (a series of the form $\sum_{i=1}^{N} \gamma_{i} e^{-t / \tau_{i}}$ ) representation of the material, which is further described below. 


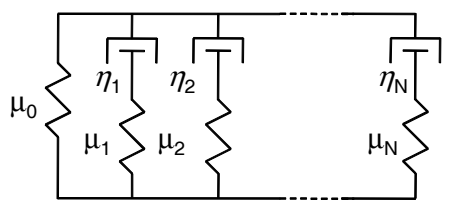

Figure 2: General Maxwell element with $\mathrm{N}$ components.

The stress is decomposed into hydrostatic and deviatoric components in order to isolate the viscoelastic effect from the volumetric terms:

$$
\boldsymbol{\sigma}^{n+1}=\kappa \operatorname{tr} \boldsymbol{\varepsilon}^{n+1} \boldsymbol{I}+\operatorname{dev} \boldsymbol{\sigma}^{n+1},
$$

where $\kappa$ is the bulk modulus, $\operatorname{tr} \varepsilon^{n+1}$ is the trace of $\varepsilon^{n+1}$, and dev $\sigma^{n+1}$ is the deviatoric part of the material stress tensor for the current $(n+1)$ time step. The deviatoric part of the stress takes on the following form:

$$
\operatorname{dev} \boldsymbol{\sigma}^{n+1}=\operatorname{dev} \boldsymbol{\sigma}_{0}^{n+1}+\sum_{i=1}^{N} \boldsymbol{h}_{i}^{n+1},
$$

where $\boldsymbol{\sigma}_{0}$ is the elastic stress tensor and $\boldsymbol{h}_{i}$ are the internal stress variables that come from the so called heredity integral:

$$
\boldsymbol{h}_{i}(t)=\int_{o}^{t} \gamma_{i} e^{-\frac{t-s}{\tau_{i}}} \frac{\partial \boldsymbol{\sigma}_{0}(s)}{\partial s} \mathrm{ds} .
$$

Splitting this integral into parts that are known (i.e., time period $\left[0, t^{n}\right]$ ) and unknown (i.e., time period $\left[t^{n}, t^{n+1}\right]$ ), and using the approximation $\frac{\partial \sigma_{0}(t)}{\partial t} \approx$ $\frac{\sigma_{0}^{n+1}-\sigma_{0}^{n}}{\Delta t}$, the internal stress variables at the next time step are approximated as follows:

$$
\boldsymbol{h}_{i}^{n+1} \approx e^{-\frac{\Delta t}{\tau_{i}}} \boldsymbol{h}_{i}^{n}+\gamma_{i} \frac{1-e^{\frac{-\Delta t}{\tau_{i}}}}{\frac{\Delta t}{\tau_{i}}}\left[\operatorname{dev} \boldsymbol{\sigma}_{0}^{n+1}-\operatorname{dev} \boldsymbol{\sigma}_{0}^{n}\right],
$$

where $\gamma_{i}$ are the normalized relaxation constants, $\tau_{i}$ are the relaxation times, and $\Delta t=t^{n+1}-t^{n}$ is the time step. Both $\gamma_{i}$ and $\tau_{i}$ represent terms of a Prony series and are determined from empirical data as described below.

Important for nonlinear finite element analyses using an implicit formulation with a Newton algorithm is the material stiffness tensor. The tensor is computed as follows for the viscoelastic material:

$$
\mathcal{C}^{n+1}=\kappa \boldsymbol{I} \boldsymbol{I}+2 \mu_{0}\left[1+\sum_{i=1}^{N} \gamma_{i} \frac{1-e^{-\frac{\Delta t}{\tau_{i}}}}{\frac{\Delta t}{\tau_{i}}}\right]\left(\boldsymbol{I}-\frac{1}{3} \boldsymbol{I} \boldsymbol{I}\right),
$$

where $\mathcal{I}$ is the fourth rank unity tensor. 


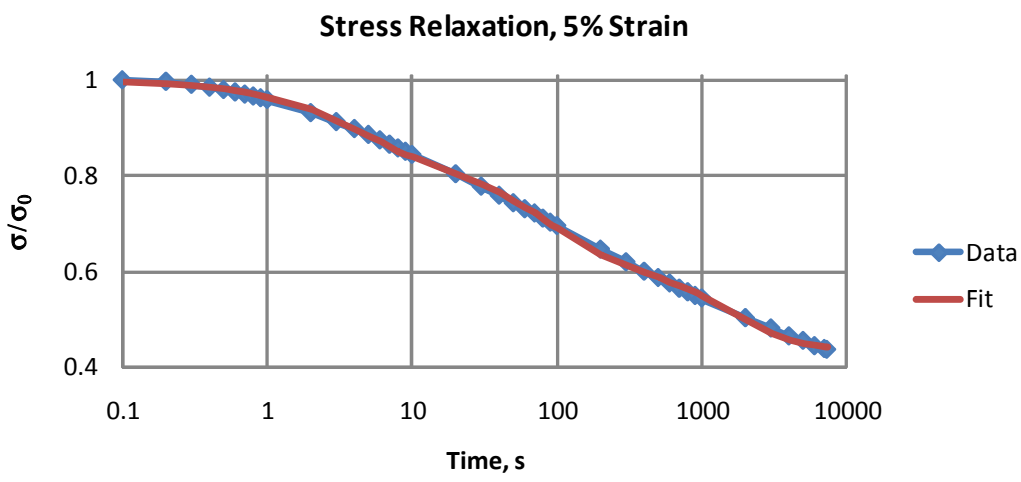

Figure 3: Stress relaxation data and the resulting Prony series curve fit.

Table 1: Prony series stress relaxation parameters.

\begin{tabular}{ccl}
\hline Component, $i$ & $\gamma_{i}$ & \multicolumn{1}{c}{$\tau_{i}$} \\
\hline 1 & 0.1484 & 4.130 \\
2 & 0.2115 & $8.195 \times 10^{1}$ \\
3 & 0.1993 & $1.610 \times 10^{3}$ \\
\hline
\end{tabular}

Relaxation constants and times, $\gamma_{i}$ and $\tau_{i}$, are determined from empirical stress relaxation data of tensile test samples. The polymeric material used in this work has a 95 A Shore hardness and is from the Hapflex line of materials purchased from Hapco, Inc., Massachusetts USA. The constants are determined by a leastsquares fit to uni-axial tensile stress relaxation data shown in figure 3 along with the resulting fit. The parameters used to create this fit are provided in table 1 . The remaining parameters required for the structural model are the long term modulus, $E_{0}$, and Poisson's ratio, $\nu$. The material is assumed to be nearly incompressible and thus $\nu=0.49$ is used in the simulations. The long term modulus has been estimated from empirical data to be $E_{0}=30.4 \mathrm{MPa}$.

The finite-element solver used for this effort has been been implemented by the authors in the form of a $\mathrm{C} / \mathrm{C}++$ program that can easily be combined and compiled with OpenFOAM. The use of a separate finite-element solver instead of the existing structural finite volume solver in OpenFOAM is a matter of preference.

\subsection{Coupling approach}

The algorithm defining the solution procedure is provided in figure 4. As indicated in this figure, a fixed-point iteration is performed with under-relaxation to ensure the fluid pressures and solid displacements are tightly converged before moving on to the next time step. 
The under-relaxation approach employs the Aitken $\Delta^{2}$ method [8] to define the dynamic relaxation coefficient. The coefficient is calculated as follows:

$$
\omega_{i}=-\omega_{i-1} \frac{\boldsymbol{r}_{i}^{T}\left(\boldsymbol{r}_{i+1}-\boldsymbol{r}_{i}\right)}{\left|\boldsymbol{r}_{i+1}-\boldsymbol{r}_{i}\right|^{2}},
$$

where $\boldsymbol{r}_{i}=\hat{\boldsymbol{u}}_{\Gamma_{F / S}, i}-\boldsymbol{u}_{\Gamma_{F / S}, i-1}$ and $\hat{\boldsymbol{u}}_{\Gamma_{F / S}, i}$ are the computed fluid/structure interface displacements for iteration $i$. The prediction of the displacements for the current iteration is then $u_{\Gamma_{F / S}, i}=u_{\Gamma_{F / S}, i-1}+\omega_{i}\left(\hat{u}_{\Gamma_{F / S}, i}-u_{\Gamma_{F / S}, i-1}\right)$, as indicated in the coupling algorithm of figure 4. The iteration stops when $\left|\boldsymbol{r}_{i}\right| / \sqrt{n}<\epsilon$, where $n$ is the length of $\boldsymbol{r}_{i}$ and $\epsilon$ is the error tolerance.

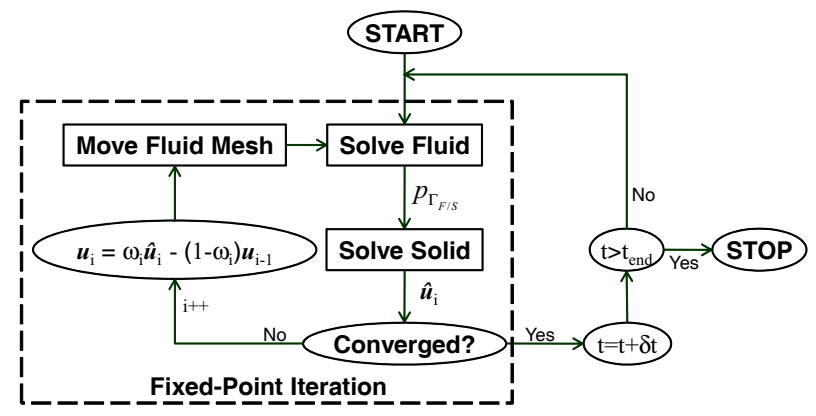

Figure 4: Implicit coupling scheme with under-relaxation.

\section{Simulation results}

The important variables to monitor for the current simulation are blade deflection and net blade load because these will be measured during the upcoming water tunnel tests. Sample results for the blade deflection, shown by blade tip sections, are provided in figure 5 . The net blade forces, from integrating the fluid pressures over $\Gamma_{F / S}$ are shown in figure 6 . This figure shows results for a baseline model and the viscoelastic model. The baseline model uses a constant constitutive relationship wherein the modulus represents the material response to an instantaneously applied load.

The blade AOA at the tip section changes from zero at the start of the simulation to increasingly negative values, reaching $-8.1^{\circ}$ at $t=360 \mathrm{~s}$. The blade lift (lift $=-F_{y}$ ) decreases to zero after about $50 s$ of operation, and then continues to decrease with time as indicated in figure 6 . This figure also shows the large difference in force magnitude between the baseline and viscoelastic models and the strong temporal dependence of the viscoelastic model. Note that the the first $360 \mathrm{~s}$ of operation are shown in this figure. Results have been computed for longer times and indicate the system is nearly at a steady state condition after approximately one hour of operation. 


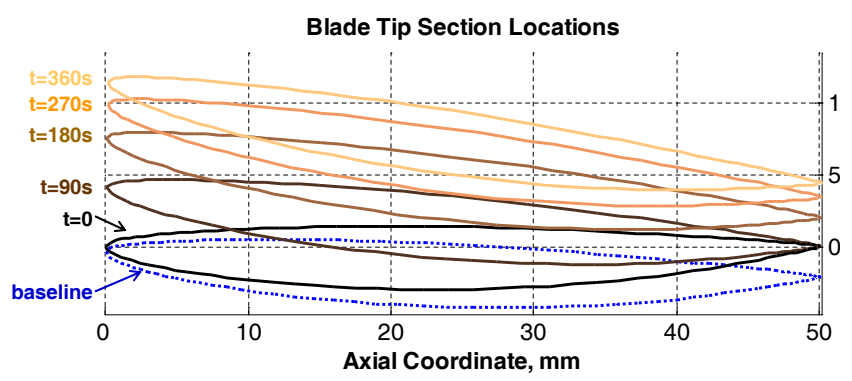

Figure 5: Blade tip section deflections; curves show results from different simulation times; baseline model response shown by dotted curve.

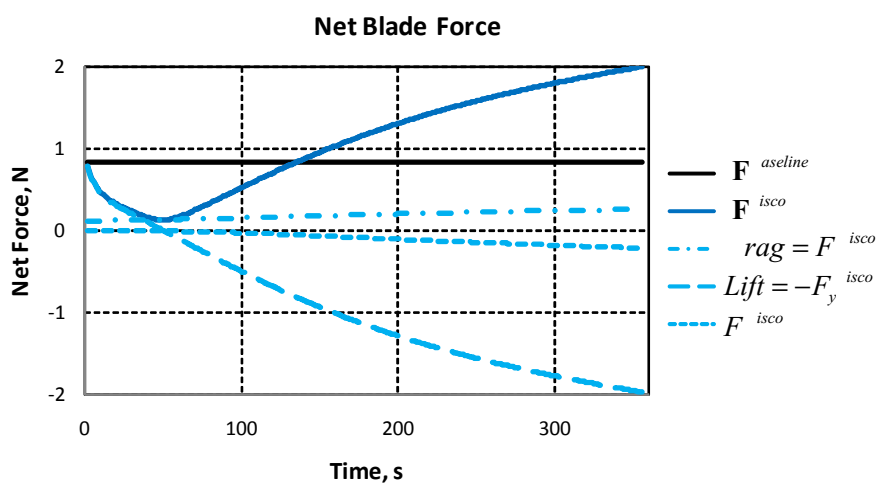

Figure 6: Net blade forces for baseline (using the instantaneous modulus) and viscoelastic models.

\section{Summary and conclusions}

Tightly coupled FSI simulations have been used for a single hydrofoil subjected to quasi-steady flow to model the effects of a viscoelastic hydrofoil operating in a water tunnel test section. The fluid domain is discretized using the finite volume approach, and solved using OpenFOAM. The flow is modeled as incompressible, laminar, and steady. The moving mesh in the fluid domain is accomplished through the tetrahedral decomposition approach with Laplace smoothing, as implemented in OpenFOAM. The solid domain is discretized with finite elements and solved using an author-written solver that is compiled with the OpenFOAM solver to facilitate data transfer necessary for the domain coupling. Simulation results for blade deformations and loads with respect to time show the dramatic effect of employing the viscoelastic constitutive relationship instead of an equivalent material modulus. The AOA 
of the blade monotonically decreases from zero to increasingly negative values (reaching $-8.1^{\circ}$ at $t=360 \mathrm{~s}$ ), with concomitant decreases in blade lift. It can be concluded that the inlet flow velocity of $2 \mathrm{~m} / \mathrm{s}$ should be satisfactory for use in the water tunnel test to provide blade deformations and forces large enough to be easily measured.

\section{References}

[1] Tezduyar, T.E. \& Sathe, S., Modelling of fluid-structure interactions with the space-time finite elements: solution techniques. International Journal for Numerical Methods in Fluids, 54(6-8), pp. 855-900, 2007.

[2] Felippa, C., Park, K. \& Farhat, C., Partitioned analysis of coupled mechanical systems. Computer Methods in Applied Mechanics and Engineering, 190(2425), pp. 3247-3270, 2001.

[3] Longatte, E., Verreman, V. \& Souli, M., Time marching for simulation of fluid-structure interaction problems. Journal of Fluids and Structures, 2008.

[4] Brockett, T., Minimum pressure envelopes for modified NACA-66 sections with NACA $\mathrm{a}=0.8$ camber and Buships type I and type II sections. Technical Report 1780, David Taylor Model Basin, U.S. Navy, 1966.

[5] Jasak, H. \& Tuković, Z., Automatic mesh motion for the unstructured finite volume methodupt. Transactions of FAMENA, 30(2), pp. 1-20, 2006.

[6] Hibbitt, K., Sorenson. ABAQUS user's manual, version 6.6, 2006.

[7] Kaliske, M. \& Rothert, H., Formulation and implementation of threedimensional viscoelasticity at small and finite strains. Computational Mechanics, 19(3), pp. 228-239, 1997.

[8] Küttler, U. \& Wall, W., Fixed-point fluid-structure interaction solvers with dynamic relaxation. Computational Mechanics, 43(1), pp. 61-72, 2008. 